\title{
Clinical cases of amyotrophic lateral sclerosis with atypical onset concurrent with hydromyelia
}

\author{
Enver Bogdanov ${ }^{1}$, Elena Mendelevich ${ }^{1}$, Aydar Khabibrakhmanov ${ }^{1}$, Shamil Bogdanov ${ }^{1}$, \\ Gyulnara Mukhamedzhanova ${ }^{1}$, and Marat Mukhamedyarov ${ }^{1}$ \\ ${ }^{1}$ Kazan State Medical University
}

May 27, 2020

\begin{abstract}
We present clinical cases of ALS, accompanied by hydromyelia, where we tried to find out the causes of the cavities and its connection with the disease. Usually, MRI does not reveal any spinal cord alterations in ALS patients. We did not find any observable cause for cavity formation.
\end{abstract}

\section{Key Clinical Message}

Accidentally detected asymptomatic hydromyelia in patients with neurological diseases can lead to a delay and cause complexity in making the correct diagnosis, as happened with our patients with ALS. ALS can be accompanied by hydromyelia.

\section{Introduction}

Amyotrophic lateral sclerosis is progressive absolutely fatal neurodegenerative disease that affects the upper and lower motor neurons. Disease begins with focal weakness and spreads to involve most muscles, including the diaphragm. Usually death occurs due to respiratory failure in 3 to 5 years [1]. Despite excessive researches on ALS biomarkers, diagnosis is based on clinical presentation and supported by EMG [2]. MRI basically is used to exclude ALS mimics, which helps in diagnosis.

We present clinical case of 36 years old male patient suffering ALS for 6 years with accidentally revealed slit-like syrinx cavity and looking as dilated central canal of the thoracic spinal cord, and clinical case of 61 years old female patient with ALS and dilates central canal of the cervix spinal cord. In the presented cases, patients with ALS were diagnosed with hydromyelia using MRI even before the typical fulfill clinical manifestation of ALS has developed, which led to a delay and difficulty in making a correct diagnosis. Such combination of ALS and hydromyelia has not been reported before. Here we discuss the differential diagnosis and suppose the causes of the central canal dilation in our cases.

\section{Case 1 history, examination}

In 201330 years old male patient developed a right calf muscle crampy. Already in 2014 patient developed right leg weakness, fasciculations in right leg muscles, shin atrophy, right foot drop. At that time the neurological examination revealed the muscle strength in the left limbs $5 / 5$ points, in the right limbs $5 / 5$ points, except for the extensors of the foot - where it was $2 / 5$ points; amyotrophy of the right shin muscles, the absence of sensation disturbances (Fig.1). Disease progression was slow and in 2015 amyotrophy involved right thigh muscles. By the end of 2015, the patient developed fasciculations throughout the body, painful cramps and muscle weakness in the left shin. During this time, the examination revealed brisk knee reflexes, the Babinsky sign on the right side. In 2016 patient developed left leg amyotrophy, in 2017 left foot drop. In 2018 weakness involved left thigh and upper extremities. 
The patient was observed in dynamics and in 2019 neurological assessment revealed restricted movements mostly in lower limbs, excessive amyotrophy of the right and left legs, but no observable amyotrophy of the upper limbs (Fig.2). Generalized fasciculations were noted. Motor examination showed excessive muscle weakness of the lower limbs more pronounced in distal part, and mild weakness of the upper limbs. Deep tendon reflexes were brisk with wide spread, pathological signs on upper limbs, Achilles reflex and Babinsky sign absent on both sides. Sensation to pinprick, vibration and proprioception were normal. There were no speech, swallowing, and breathe disturbances.

During the disease course the patient underwent MRI and EMG. In 2015 MRI of the spinal cord showed slitlike syrinx cavity and looking as dilated central canal of the thoracic spinal cord at the ThIII - ThIX vertebrae level, no Chiari malformation or other extramedullary factors predisposing to syringomyelia (Fig.3). MRI of the brain showed no pathology. In 2015 EMG showed signs of damage to the lower motor neurons of the of the lumbar and cervic spinal cord (both motor unit remodeling and denervation), and no conduction blocks. In 2016 EMG showed the negative trend of damage. Follow-up imaging in both 2016 and 2019 showed no significant changes in cavity size (Fig.4). Also the patient was tested for the titer of GM-1 and GM-2 antibodies (markers of multifocal motor neuropathy), which was low. Other laboratory tests of blood and CSF were normal.

\section{Discussion}

The patient's initial neurological syndrome was atrophy of the right leg. The first diagnosis was made between monomelic amyotrophy, spinal muscular atrophy, progressive muscular atrophy, and multifocal motor neuropathy. MMA is characterized by signs of spinal cord atrophy and vertebral canal stenosis, which was not detected on MRI, as well as the absence of sensitive disturbances [3]. SMA is characterized by symmetrical atrophy, when patient suffered amyotrophy and muscle weakness only in right leg for long time. EMG revealed no conduction blocks, and there was low titer for GM-1 and GM-2 antibodies, which made it possible to exclude MMN [4]. As the disease progressed, symptoms of upper motor neuron damage developed, such as brisk reflexes and pathological signs. EMG showed signs of damage to the motor neurons of the anterior horns of the spinal cord in the segments outside the affected right leg. Finally, in 2015 the combination of LMN and UMN clinical signs of one lumbar region, EMG LMN signs in lumbar and cervic regions, made it possible to diagnose clinically probable laboratory supported ALS according to the revised El-Escorial criteria concurrent with hydromyelia [2]. In 2019 the diagnosis was changed to possible ALS.

We have wondered whether the revealed central canal dilation has a clinical manifestation in this patient. Our clinical experience allows us to state that the dilation of the central canal with such small diameter without increasing over time cannot cause progressive severe paresis and atrophy of the lower limb [5].

The origin of the central canal dilation is unclear. The MRI showed no Chiari malformation or other extramedullary factors predisposing to syringomyelia. In addition, there was no history of spinal cord trauma, inflammatory processes, infarction or hemorrhage. We suppose that the presence of hydromyelia is a casual association. For example, Holly and Betzdorf (2002) argued, that slit-like syrinx cavities are remnants of the central canal and can be found in a small percentage of adults [6]. Patients with slitlike syrinx could be neurologically intact or just report the pain in different distributions. Petit-Lacour et al. (2000) demonstrated the incidence of asymptomatic slit-like syrinx could reach approximately $1.5 \%$ [7]. Follow-up imaging reveals no changes in cavity size.

However, we have found some case reports of neurodegenerative diseases concurrent with hydromyelia. In one case, Masciullo et al. (2016) described a patient with SPG56 - rare form of hereditary spastic paraplegia, caused by mutations in CYP2U1 and concurrent with hydromyelia [8]. The authors proposed that the presence of hydromyelia is not a casual association, but it may be part of the phenotype of SPG56. The authors supposed the hydromyelia in these cases is linked to mitochondrial dysfunction. It supported by the cases of hydromyelia described in patients with CMT2A and harboring mutations in MFN2 [9]. In the study Bombelli F. et al. have found an involvement of the CNS in the pathological process in these patients with CMT2A: $26 \%$ of patients develop CM abnormalities, $9 \%$ of patients develop hydromyelia. The authors also 
believe that the spinal cord cavity in these patients is not a coincidence, but is part of the phenotype. Both CYP2U1 and MFN2 encode enzymes regulating energy metabolism and mitochondrial dynamics. In recent ALS reviews mitochondrial dysfunction is indicated as one of the pathogenic links [10].

\section{Case 2 history, examination}

Female 61 years old patient was admitted to our neurology department in 2019. Patient's complains consisted progressive muscle spasticity and weakness more prominent in lower limbs, muscle atrophy, speaking and swallowing disturbances. First symptoms developed in 2012 when patient noticed walking difficulty due to spasticity and weakness in the right leg. In 3 months muscle weakness involved the left leg. The disease progressed slowly, and patient had to use a walking stick in 2014, and then a wheelchair in 2016. In 2014 hypotrophy of the interosseous muscles of the hands, and thenar hypotrophy were noted. Within the period 2012 to 2018 the disease progression was slow and included only worsening low limbs muscle weakness and spasticity. During 2014-2016 the patient had urge urinary incontinence. In 2018 muscle weakness involved the right hand, in 2019 muscle weakness involved the left hand and bulbar muscles (dysphonia, dysphagia).

In 2019 neurological examination revealed tetraparesis with severe muscle weakness in lower limbs up to $2 / 5$ points and mild weakness in upper limbs up to $4 / 5$ points. The patient was not able to stand or walk by herself. Tendon reflexes were brisk with wide spread. Rare fasciculations in limbs, pronounced tongue fasciculations, moderate muscle atrophy of the all limbs, speaking and swallowing disturbances, pathological signs, and spasticity more prominent in lower limbs were noted. There were no sensation disturbances.

Across the disease course the patient underwent MRI several times, EMG, and TMS. In 2012-2013 MRI of the lumbar spine revealed the spinal cord without any pathology. MRI of the cervic and thoracic spine revealed dilated central canal at the C6-Th1 vertebrae level and slightly narrow of vertebral canal (vertebral canal is less than vertebrae's front-rear size) in 2014. The spinal cord anterior horns damage signs in form of both motor unit remodeling and denervation were revealed by EMG in 2014 and 2015. Also in 2015 TMS showed motor pathway dysfunction at the cortical level. In 2019 dilated central canal of the cervical spinal cord remained in the same size (C6-Th1) by MRI (Fig.5). Laboratory tests of blood and CSF were normal.

\section{Discussion}

This case complements our first clinical case of a 36-years male patient suffering from ALS with a dilated central canal of the thoracic spinal cord. Both clinical cases share such features as the slow progression of the disease, with manifestation in the lower limbs, and the presence of a cavity in the spinal cord. At the same time, these cases differ from each other in their initial main leading syndrome. When in the first case the initial syndrome is lower monoparesis with the atrophy, in the second - spastic lower paraparesis. Spastic paraparesis is a condition requiring differentiation with a wide range of pathologies, including spondylogenic myelopathy, demyelinating disorders (multiple sclerosis, neuromyelitis optica), vascular diseases (spinal cord infarction, arteriovenous shunts) and neurodegenerative diseases (hereditary spastic paraplegia, primary lateral sclerosis), etc. [11].Spondylogenic myelopathy, demyelinating and vascular disorders were excluded by the brain and the spinal cord MRI that did not show any typical sign. Our patient developed symptoms (spastic paraparesis and urinary urgency) matching uncomplicated HSP except for sensory signs. PLS is the diagnosis of exclusion and general features include the clinical presence of upper motor dysfunction most commonly in the legs, and the absence of sensory signs, marked fasciculation and muscle atrophy. EMG is usually normal, but can reveal minimal denervation that does not fulfill El Escorial criteria [12].

Our patient developed notable limbs amyotrophy, marked tongue fasciculation, and EMG data met El Escorial criteria, therefore HSP and PLS were excluded. Given all the data in 2015 clinically probable laboratory supported ALS was diagnosed according to revised El Escorial criteria [2]. It was changed to possible ALS in 2017 and definite ALS in 2019.

In this case, the question of the cause of the cavity also arises. In the first case, we decided that the most likely one is just a coincidence. This patient has signs of narrowing of the spinal canal on MRI (vertebrae's front-rear size is less than size of vertebral canal). There is no history of the spinal cord damage. So we 
suppose the origin of central canal dilation is slightly narrow of vertebral canal, and this cavity can be classified as noncommunicating central canal dilation [13]. Also we suppose that such small cavity does not have a contribution in clinical manifestation. During 2014-2019, the cavity remained at the same size, despite a dramatic clinical deterioration in the last 2 years.

In the literature we have not met reports of cases of the ALS with hydromyelia, it has to be a very rare combination. But taking into account the fact that hydromyelia is a phenotypic feature of some neurodegenerative diseases $[8,9]$, there is the possibility that hydromyelia may be a part of the phenotype in the rare cases of ALS. Further observations are required.

\section{Author contribution}

E.I. Bogdanov, E.G. Mendelevich, A.N. Khabibrakhmanov, G.R. Mukhamedzhanova were attending doctors in the case 1. E.I. Bogdanov, A.N. Khabibrakhmanov were attending doctors in the case 2. E.I. Bogdanov and E.G. Mendelevich conceived the study. G.R. Mukhamedzhanova collected case 1 data in 2015 - 2016. A.N. Khabibrakhmanov collected case 1 and 2 data in 2019, prepared the original draft. E.I. Bogdanov, S.E Bogdanov, and M.A. Mukhamedyarov - draft review and editing, instrumental data analyzing. All authors read and approved the final manuscript.

\section{References}

1. Zarei S. et al. 2015. A comprehensive review of amyotrophic lateral sclerosis. Surgical Neurology International 16 (6):171. doi: 10.4103/2152-7806.169561

2. Ludolph A, Drory V, Yardiman O, et al. 2015. WFN Research Group On ALS/MND A revision of the El Escorial criteria - 2015. Amyotroph Lateral Scler Frontotemporal Degener 16(5-6):291-292. doi: 10.3109/21678421.2015.1049183.

3. Gourie-Devi M. 2007. Monomelic amyotrophy of upper or lower limbs. Handbook of Clinical Neurology 82:207-227. doi: 10.1016/S0072-9752(07)80014-5.

4. Van Schaik IN, Bouche P, Illa I, et al. 2006. European Federation of Neurological Societies. Peripheral Nerve Society guideline on management of multifocal motor neuropathy. Eur J Neurol 13(8):802-808. doi: 10.1111/j.1468-1331.2006.01466.x

5. Bogdanov EI, Mendelevich EG, Ibatullin MM. 2000. Spontaneous drainage in syringomyelia: magnetic resonance imaging findings. Neuroradiology 42(9):676-678.

6. Holly L.T. and Batzdorf U. 2002. Slitlike syrinx cavities: a persistent central canal. Journal of Neurosurgery 97(2):161-165. doi: 10.3171/spi.2002.97.2.0161

7. Petit-Lacour M.C. et al. 2000. Visibility of the central canal on MRI. Neuroradiology 42(10):756-761. doi: $10.1007 / \mathrm{s} 002340000373$.

8. Masciullo M., et al. 2016. Hereditary spastic paraplegia: novel mutations and expansion of the phenotype variability in SPG56. European Journal of Paediatric Neurology 20(3):444-448. doi: 10.1016/j.ejpn.2016.02.001.

9. Bombelli F. et al. 2014. Charcot-Marie-Tooth disease type 2A: from typical to rare phenotypic and genotypic features. JAMA Neurology 71(8):1036-1042. doi: 10.1001/jamaneurol.2014.629.

10. Carrì MT, D'Ambrosi N, Cozzolino M. 2017. Pathways to mitochondrial dysfunction in ALS pathogenesis. Biochem Biophys Res Commun 483(4):1187-1193. doi 10.1016/j.bbrc.2016.07.055.

11. Souza P, Pinto W, Batistella G, Bortholin T, Oliveira A. 2016. Hereditary Spastic Paraplegia: Clinical and Genetic Hallmarks. The Cerebellum 16(2). doi: 10.1007/s12311-016-0803-z.

12. Statland JM, Barohn RJ, Dimachkie MM, Floeter MK, Mitsumoto H. 2015. Primary Lateral Sclerosis. Neurol Clin 33(4):749-760. doi: 10.1016/j.ncl.2015.07.007. 
13. Milhorat T.H., Fox A., Todor D.R. Pathology, Classification, And Treatment of Syringomyelia. In: Tamaki N., Batzdorf U., Nagashima T. (eds) Syringomyelia. Springer, Tokyo. 2001. Doi: 10.1007/978-4-431-678939_2.

\section{Figure legend}

Figure $1-2015$, atrophy of the only right leg, most prominent in distal part.

Figure 2 - 2019, atrophy involved the left leg.

Figure 3 - 2015, T2-weighted MRI of thoracic spine, anteroposterior size $6.75 \mathrm{~mm}$, width $7.74 \mathrm{~mm}$, cavity size $1.87 \mathrm{~mm}$ (anteroposterior size and width lines are offset to avoid overlapping).

Figure 4 - 2019, T2-weighted MRI of thoracic spine, anteroposterior size $6.53 \mathrm{~mm}$, width $7.27 \mathrm{~mm}$, cavity size $2.02 \mathrm{~mm}$ (anteroposterior size and width lines are offset to avoid overlapping).

Figure 5 - 2019, T2-weighted MRI shows dilated central canal at the C6-Th1 vertebrae level and slightly narrow of vertebral canal (vertebral canal is less than vertebrae's front-rear size). (Unfortunately the patient was unable to provide previous MRI from 2014 and 2018)

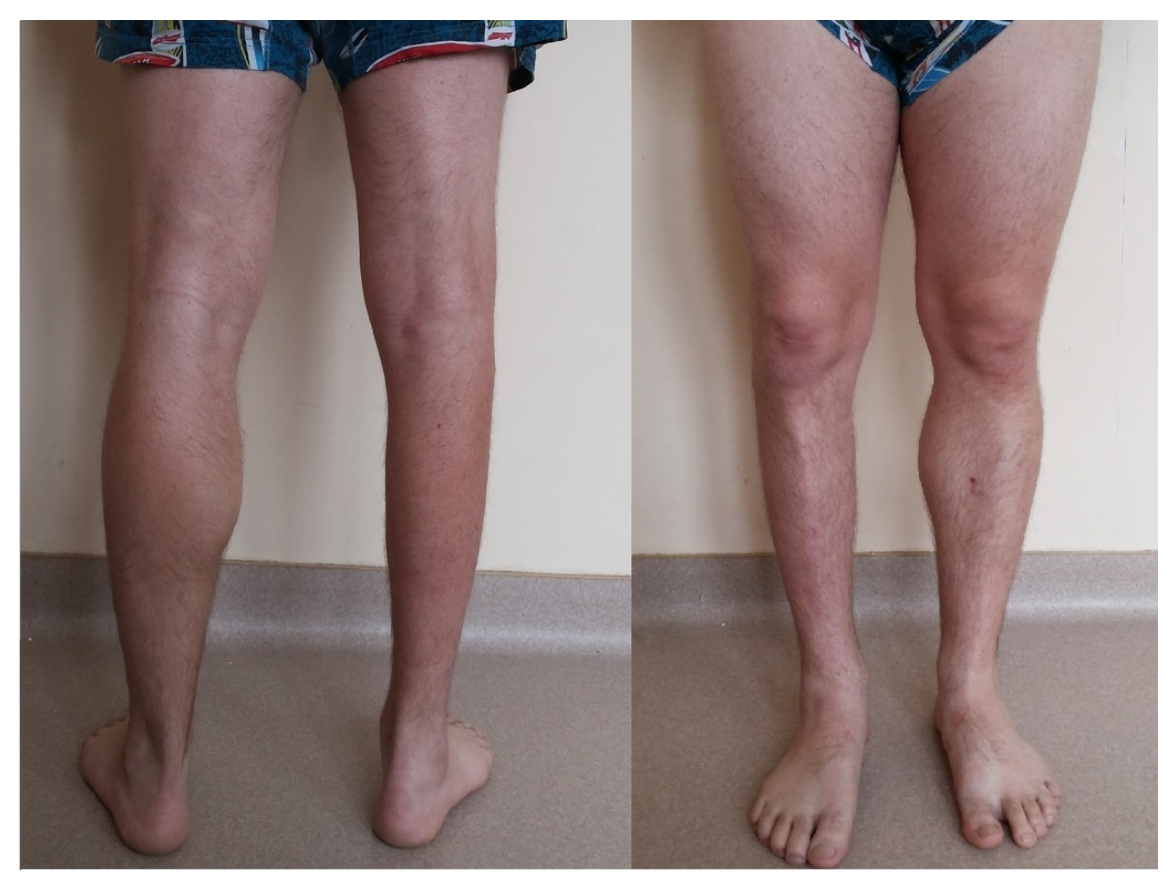






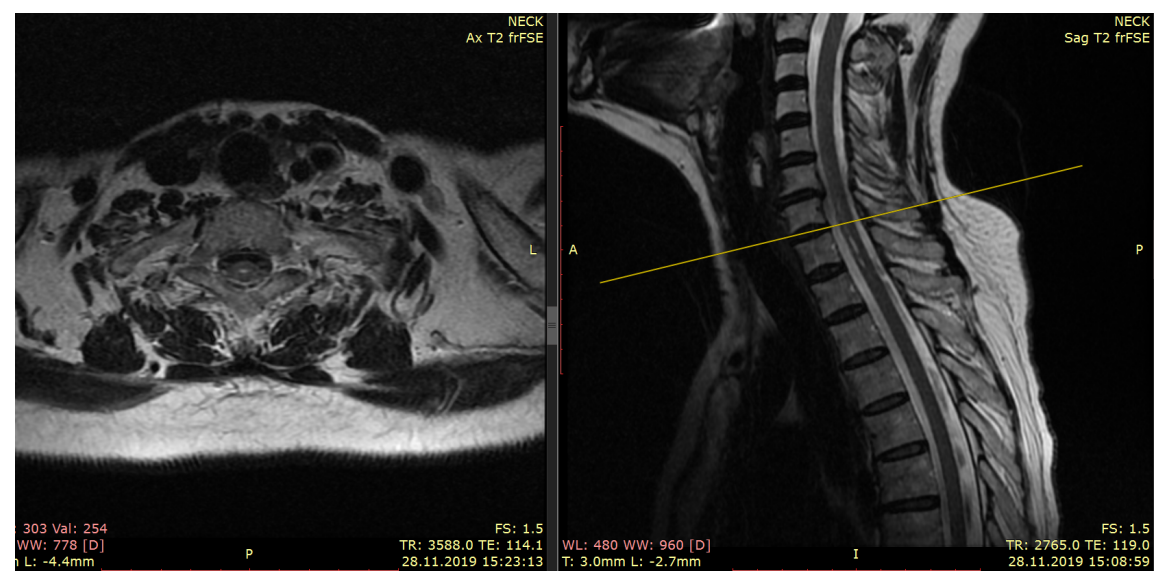

